\title{
Declared and actual students' physical activity
}

\author{
Jarosław Cholewa ${ }^{1 \mathrm{ABD}}$, Miłosz Witkowski ${ }^{\mathrm{BC}}$, Jacek Wąsik ${ }^{3 \mathrm{AD}}$, Tomasz Góra ${ }^{3 \mathrm{AD}}$ \\ ${ }^{1}$ The Jerzy Kukuczka Academy of Physical Education in Katowice, Poland \\ ${ }^{2}$ State University of Applied Sciences in Racibórz, Poland \\ ${ }^{3}$ Jan Dlugosz University in Częstochowa, Poland
}

Authors' Contribution: A - Study design; B - Data collection; C - Statistical analysis; D - Manuscript Preparation; E - Funds Collection

\begin{abstract}
Purpose: $\quad$ Regular and reasonable physical activity seems to be one of the most recommended health-promoting human behaviours. The aim of the work was to determine the relationship between the declared and actual level of students' physical activity.

Material: $\quad 118$ students from the faculty of physical education aged $22.62 \pm 1.93$ years participated in the study ( 61 men aged $23.12 \pm 1.78$ years and 57 women aged $22.08 \pm 1.39$ years). To determine the level of physical activity, the International Physical Activity Questionnaire (IPAQ) and the three-axis ActiGraph GT3X accelerometer were used in order to record physical activity parameters and the amount of energy expenditure.

Results: $\quad$ The largest absolute difference without gender division was found in the average duration of physical activity in the medium intensity zone (16.83\%). Differences in the level of weekly level of physical activity were noted in the whole group $(13.79 \% ; p<0.01)$ as well as in the group of women (relative difference $18.07 \%, p<0.01$ ) and in the group of men $(9.74 \%, p<0.01)$. A high correlation was observed between weekly energy expenditure calculated on the basis of declared values and the actual energy expenditure $(r=0.94 ; p<0.05)$.

Conclusions: $\quad$ Research carried out as part of the study showed that students declare a higher level of physical activity compared to the actual performed activity. On this basis, it can be concluded that there is a risk of error in assessing the volume of physical activity in a survey. The study showed significant gender diversity in the frequency and duration of physical activity undertaken by the respondents.

Keywords: physical activity, IPAQ, accelerometer, physical education, students.
\end{abstract}

\section{Introduction}

The shortage of physical activity of modern generations is one of the reasons for the increase in the frequency of diseases of the circulatory and respiratory systems, musculoskeletal system and metabolic and cancer diseases [1-4]. Regular and reasonable physical activity (PA) therefore becomes one of the most recommended health-promoting human behaviours. Determining the level of PA provides data to develop and improve theoretical models of behaviours that allow the development of specific postulates for the promotion of physical activity. They also help identify parts of the population at particular risk of hypokinesia [5, 6].

In epidemiological studies, the most commonly used method for estimating the level of physical activity is a diagnostic survey, and the measuring tools are questionnaires [7, 8]. One of the most popular is the International Physical Activity Questionnaire (IPAQ) [9, 10]. Its advantages are simple research procedures and a short time of completing the questionnaire, in which the respondents self-assess the frequency and duration of weekly physical activity in low, moderate and high intensity zones during the day and week. Globally unified survey procedures developed by the International IPAQ Committee allow its use, for comparative purposes, on all continents $[11,12]$.

Numerous comparative studies of physical activity in

(c) Jarosław Cholewa, Miłosz Witkowski, Jacek Wąsik,

Tomasz Góra, 2020

doi:10.15561/20755279.2020.0302 the European Union countries have been conducted with the use of IPAQ [7, 13]. The results of assessing the level of PA of the respondents, presented in accordance with the IPAQ protocol, are diverse and often very optimistic $[11,14]$. Some studies emphasise that this may be due to a tendency to overestimate the self-assessment of PA, especially among adolescents. There are also suggestions that the criteria for high and moderate PA levels adopted in the IPAQ protocol are probably too liberal [9]. It is emphasised that the criteria for high and moderate (average) level of weekly PA volume according to IPAQ also include low-intensity physical activities [15].

Accurate diagnosis of PA is necessary, among other things, to monitor and control the energy balance [16, 17]. Precise assessment of the intensity of physical activities allows to create targeted therapeutic programmes for people with various health problems [18]. Another advantage of monitoring physical activity is providing feedback on the level of PA being undertaken, which on the one hand allows for interpretation in relation to the recommendations of health enhancing $\mathrm{PA}$, and on the other is a motivating factor for taking up physical exercise $[2,19]$.

Among others, tests using accelerometers feature high accuracy in measuring PA [20, 21]. They are devices that record linear and angular accelerations during movements of the whole body or its parts, using electromechanical, optical or piezoelectric motion sensors. It is pointed out that most accelerometers record accelerations in 
movements of the whole body with high accuracy [22, 23]. They are adapted to be worn on the trunk and upper and lower limbs. Depending on the form of the activity being measured, they record the acceleration of the body or its parts, the number of steps taken, distance travelled, time of sitting and lying down, walking time, number of posture changes (from lying to sitting, from sitting to standing). Thanks to the software included, it is possible to archive the collected data.

Given the importance of the accuracy of measuring the level of physical activity, the purpose of the work was to determine the relationship between the declared and actual level of physical activity of students in a week of the academic year. The following research questions were formulated:

1. How do students of the physical education faculty assess the level of their physical activity?

2. Is there gender diversity in the frequency and duration of physical activity of the respondents?

\section{Material and Methods}

\section{Participants.}

118 students aged $22.62 \pm 1.93$ years participated in the study (61 men aged $23.12 \pm 1.78$ years and 57 women aged $22.08 \pm 1.39$ years). The respondents were the second- and third-year students of full-time studies in the Physical Education faculty $(\mathrm{n}=118)$ at the State University of Applied Sciences in Racibórz (Poland). Prior to commencing the study, formal consent was sought from participants. They were informed about test procedures and voluntarily participated in data collection. The study was conducted with ethic rules based on Helsinki Declaration

\section{Research Design.}

To determine the level of physical activity, the International Physical Activity Questionnaire (IPAQ) and the three-axis ActiGraph GT3X accelerometer (AC) were used. Numerous studies have indicated a satisfactory repeatability and validation of these research tools in the assessment of the characteristics of the physical activity of adults $[24,25]$.

Before proceeding with the study, accelerometers were prepared by entering into their memory data on the age, sex, height and weight of the subject. Subjects wore an accelerometer for 7 days at waking time, while during sleep they detached it without interrupting registration. After 7 days, the parameters of physical activity and the amount of energy expenditure were read from the accelerometer. On the day of reading the accelerometer data, the subjects completed the IPAQ questionnaire, assessing their physical activity in the previous week, during which they were wearing the accelerometer. The short version of the IPAQ questionnaire was used, containing questions about the number of days per week and the average duration in one day (min.), the intensity of physical activity undertaken by the subjects.

Based on the answers given by the respondents in the questionnaire (IPAQ), the volume of their physical activity in the zones of intensity: low, moderate and high (vigorous).
Weekly volume of physical activity was calculated in the above-mentioned intensity zones. The intensity of activity was determined by a multiple of metabolic equivalent (MET). 8.0 MET for high intensity activity (PA1), 4.0 MET for moderate activity (PA2) and 3.3 MET for low intensity activity (PA3) were adopted in accordance with IPAQ instructions. The calculation procedure consisted of multiplying the number of days, duration and metabolic equivalent separately for each intensity zone. The total weekly volume of physical activity (WPA) was the sum of weekly activities in individual zones, expressed in the unit of MET. This parameter was adopted as a measure of the declared physical activity of the subjects.

The accelerometer data was processed using the Actilife 6 (ActiGraph) software, which evaluated, among other things, caloric expenditure and duration of PA in individual zones, determined individually for each subject based on pulse rate. Cut-off values for lack of physical activity were set at $<25$ counts per 15 seconds. During the study, no additional incentives were used to motivate students to increase their level of physical activity.

In order to confront the results of the assessment of the weekly volume of physical activity in the IPAQ questionnaire with the accelerometer measurement, following the procedure presented in the works of Mahabir et al. [16] and Neilson et al. [26], MET units were converted to $\mathrm{kcal} / \mathrm{week}$.

\section{Statistical analysis.}

The basic descriptive statistics were calculated for the registered indicators, i.e. the mean and standard deviation were determined. The normality of the data distribution was checked by the Kolmogorov-Smirnov test. The significance of differences between the declared and actual weekly volume of physical activity of the subjects was assessed with the t-test. The correlation of the results of the assessment of their physical activity in the questionnaire and the accelerometer measurement was determined on the basis of the Pearson linear correlation coefficient. The statistical significance was assumed at $\mathrm{p}<0.05$.

\section{Results}

Table 1 contain the frequency and volume of daily physical activity of the subjects. Data analysis showed that, in all cases, the level of declared PA was higher than real, but not in all cases it was statistically significant. The largest absolute difference without gender division was found in the average duration of physical activity in the medium intensity zone $(16.83 \%)$. The smallest differences were found in the frequency of taking PA in the zone of low-intensity activities $(2.10 \%)$. Taking into account the gender division, it was found that in the group of women, the largest difference was found in the level of medium-intensity activities. The frequency of PA and duration of activities in this zone differed by $14.58 \%$ and $14.54 \%$, respectively. In the group of men, the biggest difference was in the duration of activities in the highintensity (18.82\%) and moderate (18.38\%) activity zones.

Figure 1 shows the intensity of the weekly level of 
physical exercise expressed in multiples of metabolic equivalent (MET) by gender. The differences in the weekly level of physical activity between the declared and the actual one was statistically significant both in the group of women (relative difference 18.07\%, $\mathrm{p}<0.01$ ) and in the group of men $(9.74 \%, p<0.01)$. Statistically significant differences were also found in the whole group $(13.79 \%$; $<<0.01)$.

Figure 2 shows the linear regression between the weekly energy expenditure calculated on the basis of the declared values and the actual expenditure. The calculations of the Pearson linear correlation coefficient $(\mathrm{r}=0.94 ; \mathrm{p}<0.05)$ showed a high correlation between the variables determined.

\section{Discussion}

Research carried out as part of the study showed that students declare a higher level of physical activity compared to the actual performed activity (13.79\%; $\mathrm{p}<0.01)$. On this basis, it can be concluded that there is a risk of error in assessing the volume of physical activity in a survey. This is confirmed by the results of studies where the energy expenditure estimated from various questionnaires was compared with the expenditure measured by a laboratory method of doubly labelled water [16]. With correlation coefficients of the results of parallel studies with these tools within the range of 0.5 - 0.7, in individual cases a significant overestimation of physical activity in the survey was found. The main reason

Table 1. Frequency and value of daily physical activity in 3 zone of intensity (min).

\begin{tabular}{lllllll}
\hline \multirow{2}{*}{ Intensity } & \multicolumn{5}{c}{ Female } & \multicolumn{5}{c}{ Male } & \multicolumn{2}{l}{ Total } \\
\cline { 2 - 7 } & IPAQ & AC & IPAQ & AC & IPAQ & AC \\
\hline \multicolumn{2}{l}{ Frequency of daily physical activity (day/week) } & & & & \\
PA 1 & $3.46 \pm 0.77$ & $3.09 \pm 0.77^{*}$ & $4.12 \pm 1.82$ & $3.98 \pm 1.75$ & $3.80 \pm 0.96$ & $3.55 \pm 0.83^{*}$ \\
PA 2 & $5.36 \pm 1.68$ & $4.87 \pm 1.59^{*}$ & $5.41 \pm 1.34$ & $5.23 \pm 2.27^{*}$ & $5.39 \pm 1.01$ & $5.06 \pm 1.85^{*}$ \\
PA 3 & $6.23 \pm 1.09$ & $6.01 \pm 1.16$ & $6.27 \pm 1.67$ & $6.12 \pm 1.86$ & $6.25 \pm 1.32$ & $6.07 \pm 0.96$ \\
\hline Daily volume of physical activity (min) & & & & \\
PA 1 & $46.37 \pm 5.21$ & $34.21 \pm 2.67$ & $51.32 \pm 6.89$ & $47.29 \pm 5.97^{*}$ & $48.93 \pm 6.28$ & $40.97 \pm 3.82^{*}$ \\
PA 2 & $103.85 \pm 11.28$ & $92.38 \pm 8.88^{*}$ & $120.35 \pm 15.38$ & $106.98 \pm 10.97^{*}$ & $112.38 \pm 19.13$ & $99.93 \pm 10.93^{*}$ \\
PA 3 & $145.72 \pm 15.97$ & $135.38 \pm 17.29$ & $157.38 \pm 13.67$ & $152.15 \pm 15.97$ & $151.75 \pm 18.02$ & $144.05 \pm 15.99$ \\
\hline
\end{tabular}

PA 1 - Physical activity of high intensity, PA 2 - Physical activity of moderate intensity, PA 3 - Physical activity of low intensity, IPAQ - Physical Activity Questionnaire, AC - accelerometer, ${ }^{*} \mathrm{p}<0.01$ (significance level).

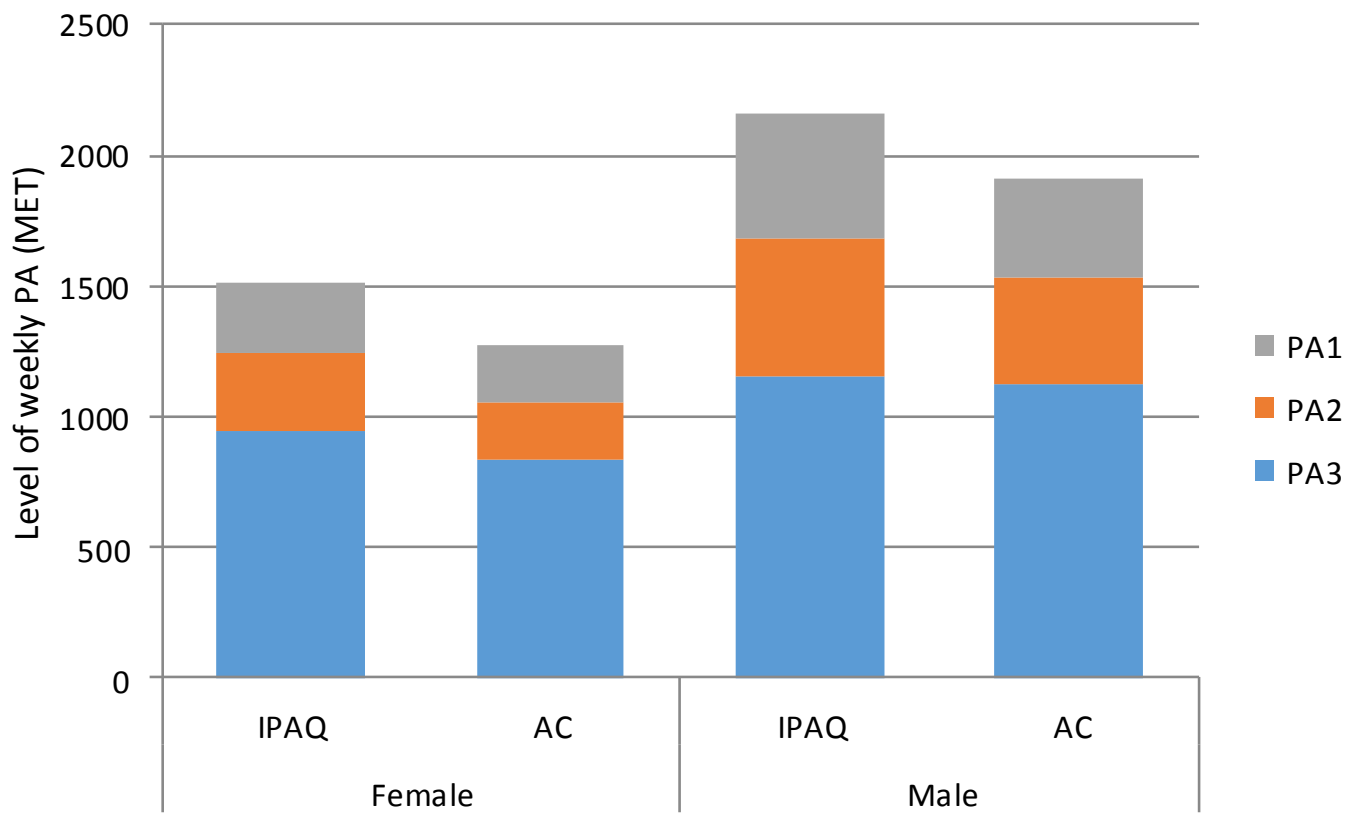

Figure 1. Level of weekly physical activity (PA) in MET: PA 1 - Physical activity of high intensity, PA 2 - Physical activity of moderate intensity, PA 3 - Physical activity of low intensity, IPAQ - Physical Activity Questionnaire, MET - metabolic equivalent, $\mathrm{AC}$ - accelerometer. 


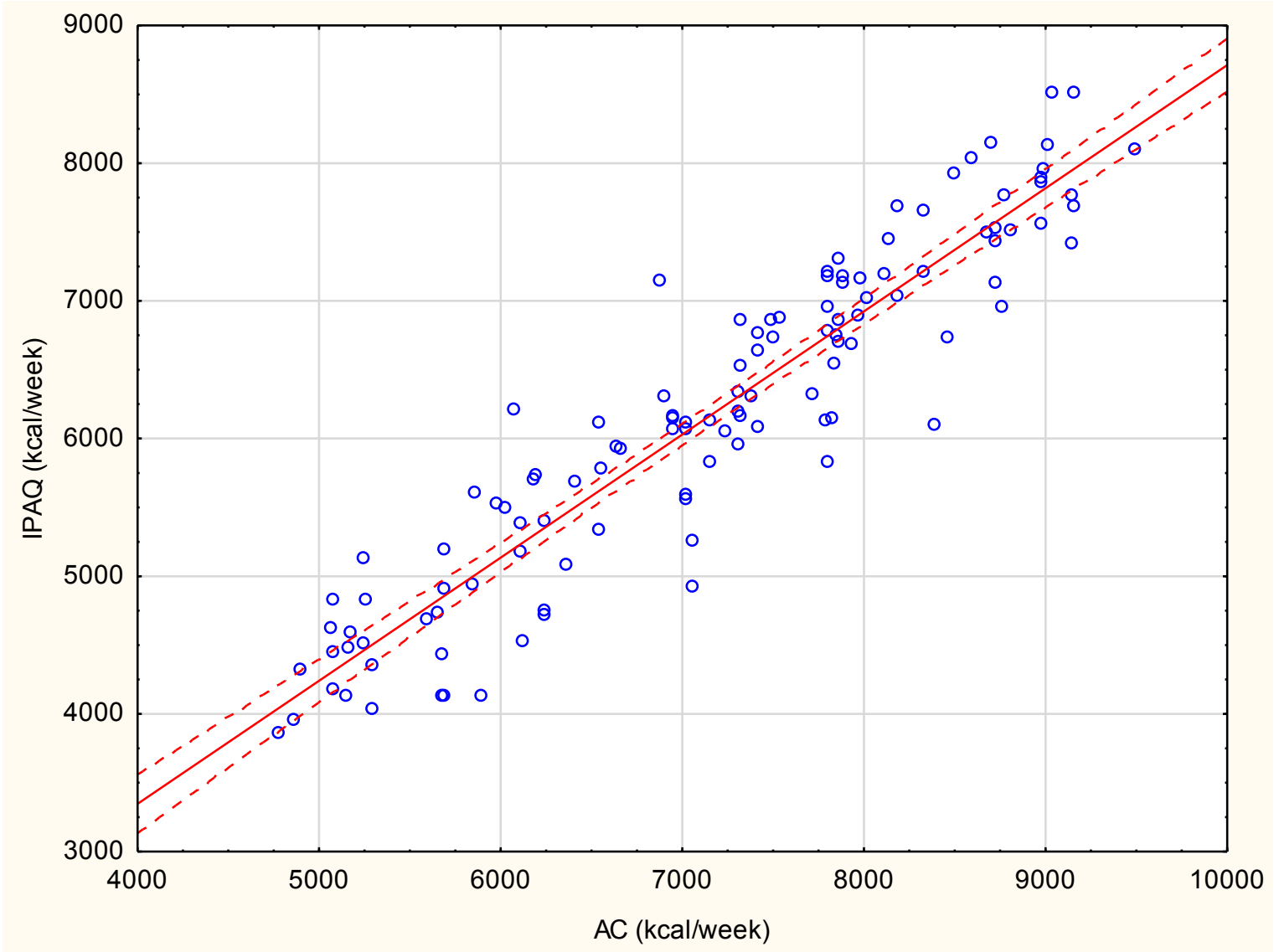

Figure 2. The relationship between the declared level of physical activity and the actual level calculated on the basis of the IPAQ questionnaire and the accelerometer (AC).

for this is the difficulty in the proper assessment of the intensity of physical activities undertaken by respondents $[16,26]$. However, considering the fact that the subjects were students of the physical education faculty, the ability to assess the intensity of physical activity should not be difficult. Perhaps these discrepancies should be explained by the tendency of respondents to overestimate their activity, especially in the zone of high and medium intensity activities [19, 27].

The above remarks refer to the average results, in individual cases the discrepancy between the selfassessment of physical activity and its measurement was significant. In a decidedly smaller number of cases a higher level of PA was found in relation to the declared activity. Therefore, in the course of further analyses, an attempt was made to verify the presented test results by assessing the strength of the correlation between the results of self-assessment of physical activity and its accelerator measurement. The high value of the correlation coefficient points to a high conformity of the self-assessment of physical activity with its measurement. Self-assessment overestimation concerns the activities taken in the respective zones [17].

The study showed significant gender diversity in the frequency and duration of physical activity undertaken by the respondents. Male students showed higher level of PA in relation to female students. This is confirmed by the results of many previous observations, in which age and sex were identified as important determinants of physical activity of the studied populations. The higher level of male physical activity and fitness compared to female is empirically confirmed [7, 12, 13].

It is emphasised in the literature that reliable diagnosis of the PA level and its assessment is a complex and multi-faceted process $[12,28]$. It should consider many elements. Among other things, the age and sex of the subjects, somatic parameters and the purpose of undertaken activity. Conducting physical activity studies in various social groups, including cross-sectional studies, gives the opportunity to characterise any differences existing in the impact of a given variable on undertaking physical activity, which may be a premise for an individualised action [11]. This problem is dealt with by, e.g., physiologists, epidemiologists, specialists in ergonomics, nutrition and also physical culture sciences. Numerous reviews of knowledge related to this subject have already been published [2, 8, 23, 28-30]. Progress in the area of knowledge in question, regarding practical implications, is significant in recent years, both in terms of measurement and diagnosis of PA. However, due to changing social conditions, knowledge of these topics requires its constant monitoring and synthesis.

\section{Conclusions}

Research carried out as part of the study showed that students declare a higher level of physical activity 
compared to the actual performed activity. On this basis, it can be concluded that there is a risk of error in assessing the volume of physical activity in a survey. The study showed significant gender diversity in the frequency and duration of physical activity undertaken by the respondents.

\section{Conflicts of Interest}

The authors declare no conflict of interest.

\section{References}

1. Grygus I. The role of physical activity in the rehabilitation of patients suffering from mild persistent bronchial asthma. Physical Activity Review, 2017; 5: 155-166. https://doi.org/10.16926/par.2017.05.20

2. Haskell WL, Lee IM, Pate RR, Powell KE, Blair SN, Franklin BA, et al. Physical activity and public health: updated recommendation for adults from the American College of Sports Medicineand the AmericanHeartAssociation. Medicine and Science in Sports and Exercise, 2007; 39(8): 1423-1434. https://doi.org/10.1249/mss.0b013e3180616b27

3. O'Donovan G, Blazevich AJ, Boreham C, Cooper AR, Crack H, Ekelund U, et al. The ABC of physical activity for health: a consensus statement from British Association of Sport and Exercise Science. Journal of Sport Science, 2010; 28(6): 573-591. https://doi.org/10.1080/02640411003671212

4. Uher I, Bukova A. Interrelationship between Exercise and Diseases in young people: Review study. Physical Activity Review, 2018; 6: 203-212. https://doi.org/10.16926/par.2018.06.25

5. Gorzkowska A, Cholewa J; Małecki A, KlimkowiczMrowiec A, Cholewa J. What Determines Spontaneous Physical Activity in Patients with Parkinson's Disease? Journal of Clinical Medicine, 2020; 9: 1296. https://doi.org/10.3390/jcm9051296

6. Jones GJ, Carlton T, Hyun M, Kanters M, Bocarro J. Assessing the contribution of informal sport to leisure-time physical activity: a new perspective on social innovation. Managing Sport \& Leisure, 2020; 25(3): 161-174. https://doi.org/10.1080/23750472.2019.1620627

7. Bergier J, Kapka-Skrzypczak L, Biliński P, Paprzycki P, Wojtyła A. Physical activity of Polish adolescents and young adults according to IPAQ: a population based study. Annals of Agricultural Environmental Medicine, 2012; 19(1): 109115 .

8. Duncan GE, Lester J, Migotsky S, Goh J, Higgins L, Borriello G. Accuracy of a novel multi-sensor board for measuring physical activity and energy expenditure. European Journal of Applied Physiology, 2011; 111(9): 2025-2032. https://doi.org/10.1007/s00421-011-1834-2

9. Biernat E, Stupnicki R, Lebiedziński B, Janczewska L. Assessment of physical activity by applying IPAQ questionnaire. Physical Education and Sport, 2008; 52:46-52. https://doi.org/10.2478/v10030-008-0019-1

10.Rodríguez-Muñoz S, Corella C, Abarca-Sos A, Zaragoza J. Validation of three short physical activity questionnaires with accelerometers among university students in Spain. Journal of Sports Medicine and Physical Fitness, 2017; 57(12): $1660-1668$

11.Drygas W, Bielecki W, Puska P. Assessment of physical activity of populations of 6 European countries under the „Bridging East-West Health Gap” Project. Polish Journal of Sport Medicine, 2002; 18(5): 169-174.

12.Rutten A, Vuillemin A, Ooijendijk WTM, Schena F, Sjostrom M, Stahl T, et al. Physical activity monitoring in Europe. The European Physical Activity Surveillance System (EUPASS) approach and indicator testing. Public Health Nutrition, 2003; 6: 377-384. https://doi.org/10.1079/PHN2002449

13.Sjostrom M, Oja P, Hagstromer M, Smith B, Bauman A. Health-enhancing physical activity across European Union countries: the Eurobarometer study. Journal of Public Health, 2006; 14: 291-300. https://doi.org/10.1007/s10389-006-0031-y

14.Rozanski A, Bavishi C, Kubzansky L, Cohen R. Association of optimism with cardiovascular events and aAll-cause mortality: A systematic review and metaanalysis. JAMA Network Open, 2019: 2(9): e1912200. https://doi.org/10.1001/jamanetworkopen.2019.12200

15.Sigmundová D, Sigmund E. The statistical and practical significance and "effect size" coefficients for the evaluation of physical activity. Physical Culture, 2012; 35(1): 55-72. https://doi.org/10.5507/tk.2012.004

16.Mahabir S, Baer DJ, Giffen C, Clevidence BA, Campbell WS, Taylor PR, et al. Comparison of energy expenditure estimates from 4 physical activity questionnaires with doubly labeled water estimates in post menopausal women. The American Journal of Clinical Nutrition, 2006; 84 (1): 230-236. https://doi.org/10.1093/ajen/84.1.230

17.Schmidt C, Santos M, Bohn L, Delgado BM, MoreiraGonçalves D, Leite-Moreira A, et al. Comparison of questionnaireandaccelerometer-basedassessmentsofphysical activity in patients with heart failure with preserved ejection fraction: clinical and prognostic implications. Scandinavian Cardiovascular Journal, 2020; 54(2): 77-83. https://doi.org/10.1080/14017431.2019.1707863

18.Cholewa J, Witkowski M, Cholewa J. Declared and real recreational physical activity with Parkinson's disease woman patients. Journal of Physical Education \& Sport, 2019; 19: 2096-2099.

19.Nawrocka A, Prończuk A, Mynarski W, Garbaciak W. Physical activity of top level managers in the context of the public health recommendations. Medycyna Pracy, 2012; 63(3): 271-279.

20.Vanhelst J, Theunynck D, Gottrand F, Beghin L. Reliability of the RT3 accelerometer for measurement of physical activity in adolescents. Journal of Sports Sciences, 2010;28(4): 375-379. https://doi.org/10.1080/02640410903502790

21.Hussey J, Bennett K, Dwyer JO, Langford S, Bell C, Gormley J. Validation of the RT3 in the measurement of physical activity in children. Journal of Science and Medicine in Sport, 2009; 12(1): 130-133. https://doi.org/10.1016/j.jsams.2007.09.010

22.Arvidsson D, Fridolfsson J, Börjesson M, Andersen LB. Ekblom Ö, Dencker M, et al. Re-examination of accelerometer data processing and calibration for the assessment of physical activity intensity. Scandinavian Journal of Medicine \& Science in Sports, 2019; 29(10): 1442-1452. https://doi.org/10.1111/sms.13470

23.Reilly JJ, Penpraze V, Hislop J, Davies G, Grant S, Paton JY. Objective measurement of physical activity and sedentary behaviour: review with new data. Archives of Disease in Childhood, 2008; 93(7): 614-619. https://doi.org/10.1136/adc.2007.133272

24.Rothney MP, Schaefer EV, Neumann MM, Choi 
L, Chen KY. Validity of Physical Activity Intensity Predictions by ActiGraph, Actical, and RT3 Accelerometers. Obesity, 2008: 16(8): 1946-1952. https://doi.org/10.1038/oby.2008.279

25.Sasaki JE, John D, Freedson PS. Validation and comparison of ActiGraph activity monitors. Journal of Science and Medicine in Sport, 2011; 14(5): 411-416. https://doi.org/10.1016/j.jsams.2011.04.003

26.Neilson HK, Robson PJ, Friedenreich CM, Csizmadi L. Estimating activity energy expenditure: how valid are physical activity questionnaires? The American Journal of Clinical Nutrition, 2008; 87(2): 279-291. https://doi.org/10.1093/ajen/87.2.279

27.Kosiba G, Gacek M, Wojtowicz A. Readiness to change and pro-health behaviours among students of physical education and other teaching specialisations. Central European Journal of Sport Sciences and Medicine, 2019: 4 (28), 53-65.

28.Pacesova P, Smela P, Stanislav Kracek S. Personal wellbeing as part of the quality of life: Is there a difference in the personal well-being of women and men with higher level of anxiety trait regarding their sport activity? Physical Activity Review, 2019; 7: 201-208. https://doi.org/10.16926/par.2019.07.24

29.Rahl RL. Physical activity and health guidelines: recommendationsforvariousages, fitnesslevels, andconditions from 57 authoritative sources. Human Kinetics. 2010. https://doi.org/10.5040/9781492595700

30.WelkGJ,McClainJJ,EisenmannJC,WickelEE.Fieldvalidation of the MTI Actigraph and BodyMedia armband monitor using the IDEEA monitor. Obesity, 2007; 15(4): 918-928. https://doi.org/10.1038/oby.2007.624

\section{Information about the authors:}

Jarosław Cholewa; Dr hab. prof. AWF; https://orcid.org/0000-0003-2179-5466; j.cholewa@awf.katowice.pl; Department of Health Related Physical Activity and Tourism, The Jerzy Kukuczka Academy of Physical Education in Katowice; Katowice, Poland.

Miłosz Witkowski; Mgr; https://orcid.org/0000-0001-5016-567X; miloszwitkowski8@gmail.com; Department of Physical Education and Health, State University of Applied Sciences in Raciborz; Raciborz, Poland.

Jacek Wąsik; Dr hab. prof. UJD; (Corresponding Author); https://orcid.org/0000-0002-6285-7283; j.wasik@ajd.czest.pl; Faculty of Health Science, Jan Dlugosz University in Czestochowa; Częstochowa, Poland.

Tomasz Góra; Mgr; https://orcid.org/0000-0002-0660-4038; t.gora@ujd.edu.pl; Faculty of Health Science, Jan Dlugosz University in Czestochowa; Częstochowa, Poland.

\section{Cite this article as:}

Jarosław Cholewa, Miłosz Witkowski, Jacek Wąsik, Tomasz Góra. Declared and actual students' physical activity. Physical Education of Students, 2020;24(3):135-140.

https://doi.org/10.15561/20755279.2020.0302

This is an Open Access article distributed under the terms of the Creative Commons Attribution License, which permits unrestricted use, distribution, and reproduction in any medium, provided the original work is properly cited http://creativecommons.org/licenses/by/4.0/deed.en

Received: 04.04.2020

Accepted: 11.05.2020; Published: 30.06 .2020 